\title{
Proton Charge Transport in Nafion Nanochannels
}

\author{
E. Valenzuela ${ }^{1, a}$ S.A. Gamboa ${ }^{2}$, P.J. Sebastian ${ }^{1,2}$, J. Moreira ${ }^{1}$, J. Pantoja ${ }^{1}$, \\ G. Ibañez ${ }^{1}$, A. Reyes ${ }^{1}$, B. Campillo ${ }^{3}$, and S. Serna ${ }^{4}$ \\ ${ }^{1}$ Universidad Politécnica de Chiapas, Cuerpo Académico de Energía y Sustentabilidad, Eduardo J. \\ Selvas S/N, Col. Magisterial, 29010, Tuxtla Gutiérrez, Chiapas. \\ ${ }^{2}$ Centro de Investigación en Energía-UNAM, 62580 Temixco, Morelos, Mexico. \\ ${ }^{3}$ Facultad de Química e Instituto de Ciencias Físicas-UNAM, Cd. Universitaria, 04510, México D.F. \\ ${ }^{4}$ Centro de Investigación en Ingeniería y Ciencias Aplicadas-UAEM, Av. Universidad 1001, 62251, \\ Cuernavaca, Morelos, México. \\ aedgarvm@gmail.com (corresponding author)
}

Received: March $26^{\text {th }}, 2008$, revised: July $12^{\text {th }}, 2008$, accepted: September $13^{\text {th }}, 2008$

Keywords: Protonic charge transport, Electrochemical impedance spectroscopy, Nafion, PEMFC.

\begin{abstract}
The Nafion perfluorinated membranes are one of the best electrolytes used in the Proton Exchange Membrane Fuel Cell (PEMFC). Some methods have been used to study the electrical properties of Nafion; nevertheless, there are some aspects of the conduction process that are not well understood, such as the contribution of the bulk and the interfacial phenomena to the total proton conduction process. In this work the Electrochemical Impedance Spectroscopy (EIS) was employed in a four electrode system to study the protonic charge transport under conditions that simulate the operation of the PEMFC. Two Nafion membranes were evaluated to determine the relation of the activation procedure with the resistance to the protonic charge transference. The results are not only consistent with other measurements but also allow to separate the protonic charge transference process in two stages. Each stage was studied and their electrical parameters were calculated using Electrical Equivalent Circuits.
\end{abstract}

\section{Introduction}

One of the major concerns of the world is to satisfy the actual energy demands required by contemporary societies. The depletion of the fossil fuels along with the collateral damage caused by pollution and climate change are leading to develop new and environmentally friendly energy sources. The Proton Exchange Membrane Fuel Cell (PEMFC) is one of the most promising solutions that could satisfy this growing energy demand and at the same time would help to preserve the environment.

In a PEMFC the hydrogen in contact with a catalyst is oxidized and as a result electrons and protons $\left(\mathrm{H}^{+}\right)$are generated. The electrons are conducted outside the cell and the $\mathrm{H}^{+}$charge are transferred to the cathode of the cell by means of a complex phenomenon in the Polymeric Membrane (PM). At the cathode the electrons, the protons and the oxygen supplied to the cell combines to produce heat and water (see Eqs. 1 and 2). In the PEMFC the energy of the fuel is converted in one stage to electrical energy, this fact leads to the high efficiency of these systems, about $40 \%$ at $80^{\circ} \mathrm{C}$ compared to $19 \%$ of an internal combustion engine [1].

$$
\begin{array}{ll}
\text { Anode } & \mathrm{H}_{2} \rightarrow 2 \mathrm{H}^{+}+2 e^{-} \\
\text {Cathode } & \frac{1}{2} \mathrm{O}_{2}+2 \mathrm{H}^{+}+2 e^{-} \rightarrow \mathrm{H}_{2} \mathrm{O}
\end{array}
$$

The major components of the PMFC are; i) Bipolar Plates (BPs), ii) Electrocatalyst and iii) Polymeric Membrane. Through the BPs the oxygen and hydrogen are conducted towards the electrocatalyst surface inside the cell where the electrochemical reactions take place. 
The polymeric membrane used in a PEMFC should accomplish some requirements as: chemical stability, impermeable to hydrogen and oxygen, operate without damage up to $150{ }^{\circ} \mathrm{C}$, and mechanical resistance. Besides, the most striking feature, is that the membrane should be charge conduction selective. The conduction selectivity implies that the electrolyte should exhibit high electrical resistance to the negative charges while having high conductivity to the positive ones [2].

Nafion is a perfluorinated membrane developed by Dupont ${ }^{\circledR}$ that due to its excellent electrical and chemical properties is one of the most extensively used electrolytes in PEMFC. The structural characteristics of Nafion is shown in Fig. 1.

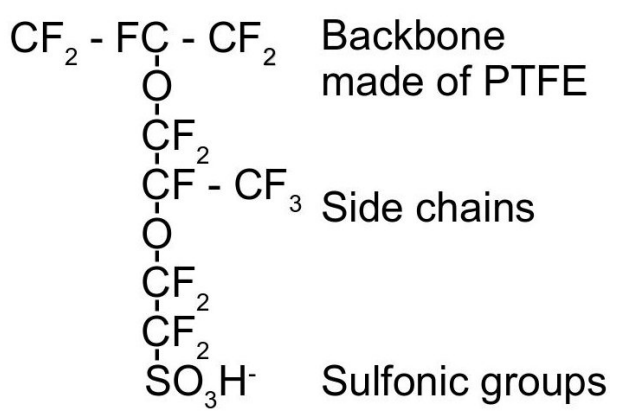

Figure 1. Nafion membrane structure; it is made of a hydrophobic backbone of PTFE with pending chains of double ether ended in $\mathrm{SO}_{3} \mathrm{H}^{-}$

Several models have been studied in order to achieve a complete understanding of the Protonic Charge Transfer (PCT) in Nafion membrane. The first approach to understanding the complex phenomena involved in the proton conduction process in Nafion are related to the study of a bactericide known as Gramicidin [3]. The Gramicidin opens a tunnel in the cellular membrane in a way that certain ions are able to enter to the bacteria cytoplasm causing an internal contamination and disease. The main disagreements with the Gramicidin and Nafion processes were that the tunnel opened by the bactericide restricts the passage of ions only according to their size, and were not able to distinguish between anions and cations [3].

Some other models were studied trying to adjust them to the extraordinary fast proton charge conduction in the Nafion; for example in the perovskites the charge of the $\mathrm{H}^{+}$ions "jumps" from one chain to the next, resulting in an apparent movement of the $\mathrm{H}^{+}$. Based on these observations a model called "Grotthuss Mechanism" was developed [4]. Despite the coincidence of this model and the phenomena observed in Nafion, some disagreements remain; for instance the distance between the sulfonic groups in Nafion is too large to allow the passage of charge from one group to the next one. Furthermore, the role of water in the proton conduction process was not elucidated. Lately, Ise [5] and Kreuer [6] based on a SAXS analysis, proposed the most accepted schematic representation of Nafion. Their results were contradictory to the inverted micelle theory of Gierke [7] and partially invalidate the Grotthuss mechanism for Nafion, hence the proton wire concept was proposed. This model suggests the conduction of $\mathrm{H}^{+}$through a chain of water molecules [8]. This approach began to establish the relation between hydration of Nafion and its conductivity. Kreuer based on these simulations suggested the formation of two new structures, the Zundel ion $\left(\mathrm{H}_{5} \mathrm{O}_{2}{ }^{+}\right)$and Eigen ion $\left(\mathrm{H}_{9} \mathrm{O}_{4}^{+}\right)[6]$. According to this, the charge transport between a structure and another was $10^{-13} \mathrm{~s}$.

The experimental and theoretical results obtained in the study of the protonic charge transfer in Nafion [5-8] had established a relation between the presence of water in the membrane with its conductivity. In addition, the discovery of the Zundel and Eigen structures and the weakening of the bonding strength between hydrogen and oxygen in water confined in nanometric spaces such as the Nafion channels, could provide a satisfactory explanation to the phenomena observed in the protonic charge transfer in Nafion membrane $[9,10]$.

Several methods for measuring resistance in PM have been used; nevertheless, these methods have disadvantages, some of them due to membrane drying, others not consider $\mathrm{H}^{+}$ions as charge carriers or additional contributions to the membrane resistance [11,12]. In this work the selected design not only measure the resistance but study the conduction process in polymeric 
membranes in the four electrodes method. In this technique the membrane is placed between two half cells containing the species of interest in aqueous solution $\left(\mathrm{H}^{+}\right.$ions $)$. In both sides of the half cells two electrodes are placed in order to apply an electric current that will switch into ionic current in the interface electrode/solution and to measure the potential across the membrane with two reference electrodes. With this procedure it is possible to ensure; i) exclusively an ionic current is flowing through the membrane and ii) the membrane is $100 \%$ hydrated during the test [13].

The aim of this work is to apply some modifications to the four electrode method in order to study the protonic charge transfer in a Nafion membrane. With these modifications the system will be easier to instrument and it will allow to measure and separate the stages of the protonic charge transfer process in order to accurately enough identify slight differences of resistance between membranes.

\section{Experimental}

\section{Design of the Evaluation System}

A four electrode system was designed in order to evaluate the proton conduction process in Nafion by Electrochemical Impedance Spectroscopy (EIS). The system consists of two half cells filled with $0.5 \mathrm{M} \mathrm{H}_{2} \mathrm{SO}_{4}$ and separated by the Nafion membrane. Two Pt grids were placed on the opposite sides of these half cells in order to apply an electronic current that will turn into an ionic current in the interface Pt/Electrolyte. In the solution, the $\mathrm{H}_{2} \mathrm{SO}_{4}$ is dissociated according to Eq. 3:

$$
\mathrm{H}_{2} \mathrm{SO}_{4} \rightarrow \mathrm{H}^{+}+\mathrm{HSO}_{3}^{-}
$$

Occasionally a further dissociation occurs (Eq. 4):

$$
\mathrm{HSO}_{3}^{-} \rightarrow \mathrm{H}^{+}+\mathrm{SO}_{3}^{2-}
$$

In both cases a negative and a positive charge carrier are obtained. Due to the Nafion conduction selectiveness only the charge of the $\mathrm{H}^{+}$ions is able to be transferred through the Membrane. In this way, a system is obtained where the membrane is continuously fully hydrated and the only charge transported by the Nafion is that derived from the $\mathrm{H}^{+}$ions, as it happens in the PEMFC. The electrical potential in both sides of the membrane is measured by placing two silver (Ag) wires as pseudo-reference electrodes. The relation between electrical potential and current will determine the impedance of the membrane. The substitution of commercial reference electrodes for Ag wires diminishes the high frequency noise, as well as the total cost, simplifying the system.

The impedance measurements were recorded with a Potentiostat/Galvanostat Solartron SI 1285. A $10 \mathrm{mV}$ sinusoidal wave with variable frequency was applied in a range from $10 \mathrm{kHz}$ to 10 $\mathrm{mHz}$. The working and counter electrode were used as current electrodes, references 1 and 2 were used as potential electrodes.

\section{Preparation of the Nafion Membranes}

The Nafion membranes were activated as follows:

1. Nafion membranes were purified in $3 \% \mathrm{H}_{2} \mathrm{O}_{2}$ at $85^{\circ} \mathrm{C}$ for $30 \mathrm{~min}$.

2. Membranes were immersed in boiling de-ionized water during $30 \mathrm{~min}$.

3. Membranes were treated in $1 \mathrm{M} \mathrm{H}_{2} \mathrm{SO}_{4}$ at $85^{\circ} \mathrm{C}$ for $1 \mathrm{hrs}$.

4. Two membranes were studied, one was kept in $1 \mathrm{M} \mathrm{H}_{2} \mathrm{SO}_{4}$ at $25^{\circ} \mathrm{C}$ during $24 \mathrm{hrs}$ and the second one was immersed in the acid for $48 \mathrm{hrs}$. The latter serves to study the effect of the protonation time 
that is directly related to the concentration of $\mathrm{SO}_{3} \mathrm{H}^{-}$ions in the membrane with the electrical resistance of the membranes.

5. The excess of acid was removed by boiling the membranes in de-ionized water for $30 \mathrm{~min}$.

6 . The Nafion membranes were stored in de-ionized water.

The four electrode method should be able to distinguish (if that is the case) slightly electrical resistance difference between the two membranes due the differences in the protonation time.

\section{Evaluation of the Membranes in the Four Electrode System}

The membrane to be evaluated was placed between two half cells and the four electrode method was implemented as described above. The working and counter electrode terminals of the Potentiostat/Galvanostate were connected to two Pt mesh that work as current electrodes and the reference electrodes $(1,2)$ to the Ag wires. The EIS measurements were obtained applying a $10 \mathrm{mV}$ sinusoidal wave in a frequency range from $10 \mathrm{kHz}$ to $10 \mathrm{mHz}$ at $25^{\circ} \mathrm{C}$. The experimental impedance data was simulated with Electrical Equivalent Circuits (EEC) using the ZVIEW software in order to obtain the values of the resistance for the protonic charge transference.

\section{Results and Discussion}

The Nyquist diagrams obtained for the membranes protonated for $24 \mathrm{hrs}$ in $\mathrm{H}_{2} \mathrm{SO}_{4}$, red dots, and 48 hrs, blue dots, are shown in Fig. 2.

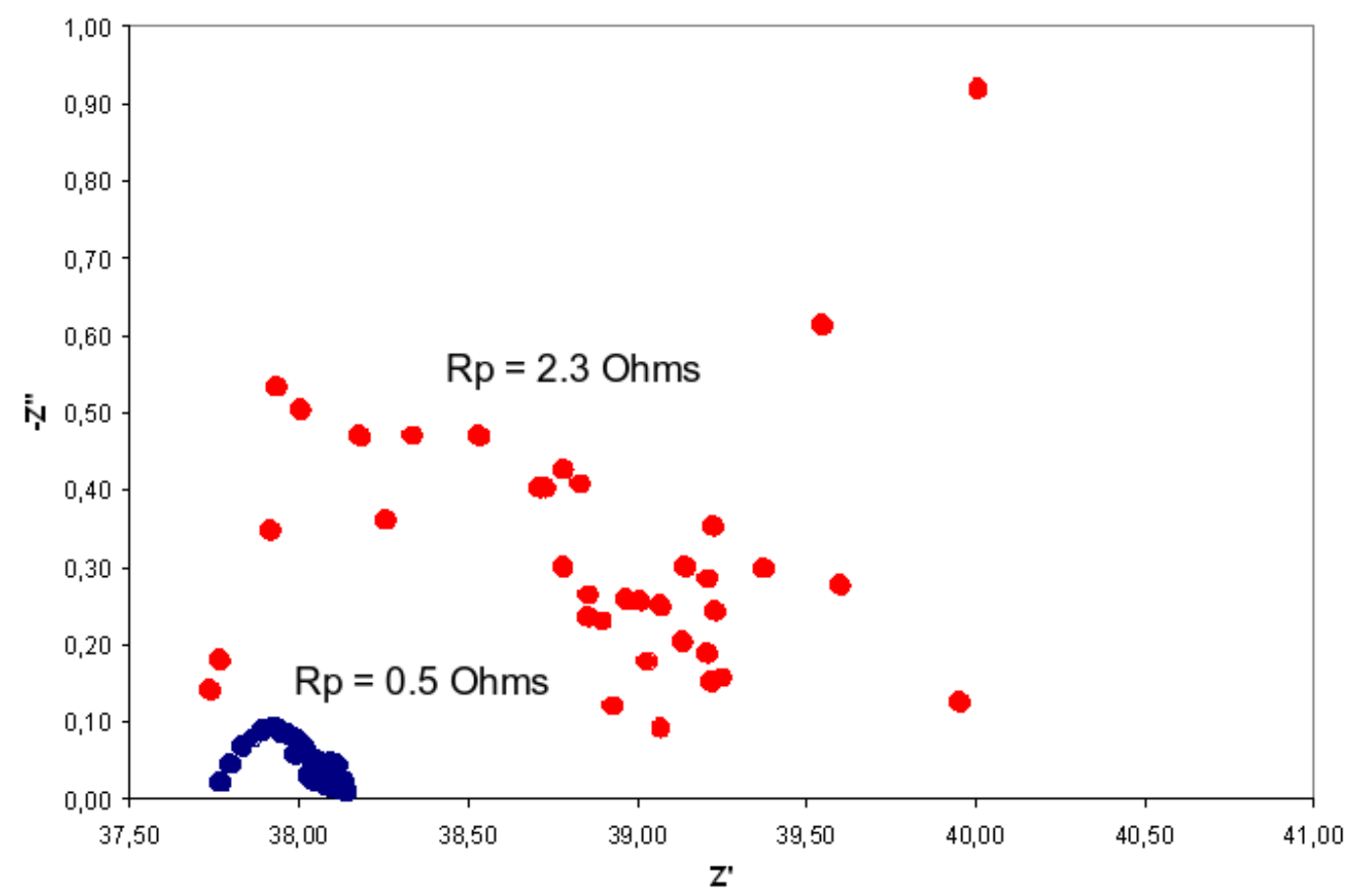

Figure 2. Nyquist diagrams for Nafion Fig. 2: The Nyquist diagrams obtained for the membranes protonated for $24 \mathrm{hrs}$ (red dot) and $48 \mathrm{hrs}$ (blue dot) in $\mathrm{H}_{2} \mathrm{SO}_{4}$.

A typical conductive membrane response was obtained; two semicircles overlapped at medium frequencies [14]. Starting at the left side of the Nyquist diagram, the first semicircle represents the process at high frequency; being these contributions attributed to the process that occurs in the bulk of the membrane. The next semicircle represents a phenomenon that takes place at low frequency; in this system, this refers to the process in the Membrane/Electrolyte interface [14]. 
The overall conduction process in Nafion can be separated in two main categories. Initially the $\mathrm{H}^{+}$charge should be transferred from the electrolyte to the membrane; this process is classified as an interfacial phenomenon, it can be related to the low frequency semicircle in the Nyquist diagram and is represented with the Diffusion Boundary Layer Resistance $\left(\mathrm{R}_{\mathrm{DBL}}\right)$ in the Electrical Equivalent Circuit (Fig. 3). After the translocation of the protonic charge from the electrolyte to the membrane, a charge transference occurs in the bulk membrane, this process is related to the high frequency semicircle and it is represented by the Heterogeneous Ionic Transfer Resistance $\left(\mathrm{R}_{\mathrm{HT}}\right)$ [15], the sum of these two process will end up with the transport of the positive charge from one side of the membrane to the opposite side.

Figure 3. The Electrical Equivalent Circuit used in the simulations

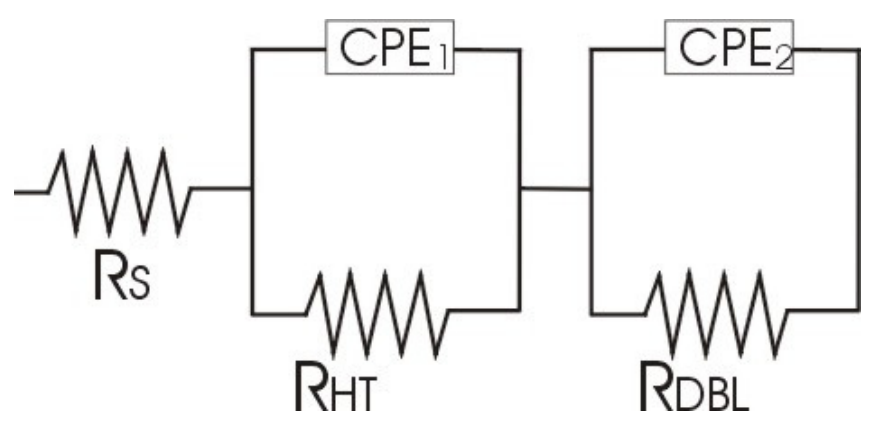

In the Nyquist diagrams in Fig. 2 the total opposition for the protonic charge transport is proportional to the length in $\mathrm{Z}^{\prime}$ axis of both semicircle radius (real impedance). Considering this, it is clear that the Nafion immersed for $24 \mathrm{hrs}$ has the higher protonic charge transfer resistance compared to Nafion immersed for $48 \mathrm{hrs}$. This result suggests a proportionate relation between the protonation time in $\mathrm{H}_{2} \mathrm{SO}_{4}$ and the conductivity of Nafion. The protonic charge resistances for the interfacial $\left(\mathrm{R}_{\mathrm{DBL}}\right)$ and for the bulk process $\left(\mathrm{R}_{\mathrm{HT}}\right)$ are presented in Table 1

Table 1. Resistance values for Nafion protonated by $24 \mathrm{hrs}$ and $48 \mathrm{hrs}$.

\begin{tabular}{cccc}
\hline Sample & \multicolumn{3}{c}{ Element } \\
\cline { 2 - 4 } Nafion 24 hrs & $\mathrm{R}_{\mathrm{HT}}(\Omega)$ & $\mathrm{R}_{\mathrm{DBL}}(\Omega)$ & Total $(\Omega)$ \\
Nafion 48 hrs & 1.3 & 1 & 2.3 \\
\hline
\end{tabular}

From Table 1 it is possible to see that the Nafion protonated for $24 \mathrm{hrs}$ has $\mathrm{R}_{\mathrm{DBL}}$ and $\mathrm{R}_{\mathrm{HT}}$ similar values. A different behavior was observed for the sample protonated for $48 \mathrm{hrs}$; in this system the $\mathrm{R}_{\mathrm{HT}}$ is considerably higher that the $\mathrm{R}_{\mathrm{DBL}}$. This response suggests that at low protonation levels the contribution of both processes are quite similar, and as the protonation level increases, the overall resistance is dominated by the process in the bulk membrane.

\section{Conclusion}

The four electrode method was used not only to measure but to study in detail the protonic charge transport in Nafion membranes. The protonic charge conductivity of the membrane is proportional to the protonation time used for the membranes preparation. The conduction process is divided in two stages, one related to the interface phenomena and the other related to the bulk membrane. The contribution of each stage of the protonic charge transport is protonation time dependant. As the protonation time increases the contribution of the bulk membrane is being predominant; this result shows that the modifications in the membrane surface such as increasing the cation concentration 
due the protonation time is closely related to the protonic charge transfer at the membrane/electrolyte interface, this characteristic could be used to study other modifications that increase the protonic charge transport in Nafion membranes.

\section{Acknowledgements}

The authors would like to thank PROMEP for the financial support for this work through the projects UPCHIS-PTC-007 and UPCHIS-PTC-020.

\section{References}

[1] P. Costamagna and S. Srinivasan, J. Power Sources Vol. 102 (2001), p. 242

[2] K.D. Kreuer, J. Membr. Sci. Vol. 185 (2001), p. 29

[3] G.V. Miloshevsky and P.C. Jordan, Biophys. J. Vol. 86 (2004), p. 92

[4] N. Agmon, Chem. Phys. Lett. Vol. 244 (1995), p. 456

[5] M. Ise, K. D. Kreuer, J. Maier, Solid State Ionics Vol. 125 (1999), p. 213

[6] K. D. Kreuer, J. Membr. Sci. Vol. 185 (2001), p. 29

[7] William Y. Hsu and Timothy D. Gierke, J. Membr. Sci. Vol. 13 (1983), p. 307

[8] Natalie Pavlenko, J. Phys.: Condens. Matter Vol. 15 (2003), p. 291

[9] N. Agmon, J. Mol. Liq. Vol. 64 (1995), p. 161

[10] S. J. Paddison, T. A. Jr. Zawodzinski, Solid State Ionics Vol. 113 (1998), p. 333

[11] T.A. Zawodzinski, M. Neeman, L.O. Sillerud, S. Gottesfeld, J. Phys. Chem. Vol. 95 (1991), p. 6040

[12] R.F. Silva, M. De Francesco, A. Pozio, Electrochim. Acta Vol. 49 (2004), p. 3211

[13] S. Slade, S.A. Campbell, T.R. Ralph, F.C. Walsh, J. Electrochem. Soc. Vol. 149 (2002), p. 1556

[14] R.F. Dalla Costa, J. Zoppas Ferreira, C. Deslouis, J. Membr. Sci. Vol. 215 (2003), p. 115

[15] J.-S. Park, J.-H. Choi, K.-H. Yeon, S.-H. Moon, J. Colloid Interface Sci. Vol. 294 (2006), p. 129 